\title{
Detection of Mass Change on a Glass Plate
}

\author{
Jannick B. Hansen - Ph.d. student ${ }^{1}$, Rune Brincker - Professor ${ }^{1}$, Manuel L. Aenlle - Lecturer ${ }^{2}$ \\ ${ }^{1}$ Department of Engineering, Aarhus University, Finlandsgade 22, 8200 Aarhus N, Denmark \\ ${ }^{2}$ Department of Construction and Manufacturing Engineering, University of Oviedo, Campus de Gijón, Zona Oeste, \\ Edificio 7, 33203 Gijón, Spain
}

\begin{abstract}
In model updating the aim is to reach the optimal correlation between FE model and test data by modifying model parameters. The traditional solution to an updating problem is obtained by non-linear optimization processes governed by the selected updating parameters and target responses. Due to imprecision and lack of information in measurements, inaccuracy in model and several possible updating parameters a wide range of potential solutions to the same problem is present.

This paper proposes a technique in which the updating problem is solved in one step using modal properties, i.e. natural frequencies and mode shapes, as target responses. The method utilizes mode shape sensitivity equations combined with the Bernal Projection equation to detect mass and stiffness discrepancies between model and experimental data. The proposed one step solution will only work accurately in cases where a reasonable FE model is available. The technique is demonstrated on simulated data of modal properties before and after mass perturbation of a glass plate. The data is polluted with noise in the range of what can be expected from real measured data.
\end{abstract}

\section{KEYWORDS: Model Updating, Damage Detection, Modal Parameters}

\section{INTRODUCTION}

Improving an FE model using test data has through many years proven to be a challenging task. There are potentially multiple sources to discrepancies between model and data and even more updating parameters. Ultimately, the solution to a specific model calibration problem is governed by parameter and target response selection and furthermore user intuition and experience. The concepts of model updating and damage detection are closely related in the sense that in both cases the aim is to obtain dissimilarity between two sets of responses. One might argue that damage detection using vibration data is model updating, limited to stiffness- and mass related parameters utilizing only the modal parameters as target responses.

The boundaries of damage detection using modal parameters reduce the amount of possible solutions. By means of mode shape and frequency sensitivity equations it is possible to obtain an estimate of damage without relying non-linear optimization processes. The proposed method detects change in structural mass and stiffness based on observed changes in modal properties, i.e. natural frequencies and corresponding mode shapes. Similar methods have with success identified structural defects by interpreting change in modal parameters [1] and the present method contributes by introducing the Bernal Projection equation [2] to calculate frequency sensitivities. The proposed method can to some extent be utilized as a model calibration tool, however, the main intent lies within the area of damage detection.

The approach is to compute mode shape and frequency sensitivities towards both mass and stiffness change. Hereafter, the location of a mass or stiffness change can be pinpointed by combining the information on the behavior of the system towards alterations with the actual observed changes in modal properties. In order to calculate mode shape sensitivities a reasonable FE model of the specific structure and the estimated modal parameters of the structure in the unperturbed or virgin state are required. In the process of calculating the frequency sensitivities the modal parameters of the perturbed system are necessary as well.

Naturally, a few aspects of the method are simplified by using simulated data. For instants, a perfect FE model is available for calculating sensitivities and there are no restrictions on the bandwidth of the "measured" data. 


\section{THEORETICAL ASPECTS}

This method utilizes both frequency and mode shape shifts as target responses to detect change in the parameters of the regarded system and is based on two equations. The initial equation is the well-known mode shape sensitivity equation [3]. The second equation is the Bernal Projection equation [2] originally intended for mode shape scaling but in this context used for determining a measure for frequency sensitivity. Both equations are featured in the final system of equations contributing to the estimate of the damage vector, i.e. the measure of change in either mass or stiffness, both location and magnitude. For brevity only the equations for detecting mass change is deduced in the following theoretical review.

\subsection{Mode Shape Sensitivity Equation}

The mode shape sensitivity equation is the first order derivative of mode shape $i$ with respect to a local change parameter $u$ in the mass and/or stiffness matrix.

$$
\frac{\partial \mathbf{b}_{i}}{\partial u}=-\frac{1}{2 m_{i}} \mathbf{b}_{i}^{T} \frac{\partial \mathbf{M}}{\partial u} \mathbf{b}_{i} \mathbf{b}_{i}+\sum_{r=1, r \neq i}^{M} \frac{1}{\omega_{i}^{2}-\omega_{r}^{2}} \frac{1}{m_{r}} \mathbf{b}_{r}^{T}\left(-\omega_{i}^{2} \frac{\partial \mathbf{M}}{\partial u}+\frac{\partial \mathbf{K}}{\partial u}\right) \mathbf{b}_{i} \mathbf{b}_{r}
$$

where $\mathbf{b}$ is a mode shape vector, $\omega$ is angular frequency, $m$ is the modal mass, $\mathbf{M}$ and $\mathbf{K}$ is the mass and stiffness matrix respectively, subscript $i$ denotes the regarded mode shape and subscript $r$ denotes the remaining mode shapes of the system. Eq. (1) can be reduced by considering a finite change in the mass matrix only. Written in matrix form

$$
\Delta \mathbf{b}_{i} \cong \mathbf{B} \boldsymbol{\Gamma}_{M, i} \mathbf{B}^{T} \Delta \mathbf{M b}_{i}
$$

where $\Delta \mathbf{b}$ is the mode change vector, $\mathbf{B}$ is the mode shape matrix, $\boldsymbol{\Gamma}$ is a diagonal matrix and subscript $M$ denotes the case of mass change. For unit mass changes in all degrees of freedom (DOF), the matching mode shape derivatives are calculated one by one. This procedure is repeated for all mode shapes. The mode shape derivatives are collected in a matrix consequently describing how the system will react to a mass change in an arbitrary DOF, this matrix will henceforth be referred to as the sensitivity matrix. The mode shape derivatives are arranged so that a multiplication with the change vector will single out the columns of the sensitivity matrix which contribute to the resulting mode shape change. In math terms

$$
\Delta \mathbf{b}_{M}=\Delta \mathbf{B}_{M} \Delta \mathbf{u}_{M}
$$

where $\Delta \mathbf{B}$ is the mode shape sensitivity matrix and $\Delta \mathbf{u}$ is the mass change vector. This vector can be determined by left multiplying Eq. (3) with the inverse of the sensitivity matrix. Due to the fact that the mode shape derivatives of all available modes are stacked in the sensitivity matrix it cannot be quadratic. Therefore, the sensitivity matrix is inverted by means of the Moore-Penrose pseudo inverse, thus

$$
\Delta \mathbf{u}_{M}=\Delta \mathbf{B}_{M}^{+} \Delta \mathbf{b}_{M}
$$

A similar expression for change in stiffness can be deduced by repeating the considerations above.

\subsection{Bernal Projection Equation}

The second equation, the Bernal Projection equation, utilizes the observed change in frequencies to detect change in either mass or stiffness in the DOF's of the system. The equation originates from research concerning scaling of mode shapes determined by operational identification techniques and reads

$$
\frac{1}{m_{i}}=\frac{\left(\omega_{b i}^{2}-\omega_{a i}^{2}\right) T_{i i}}{\mathbf{b}_{i}^{T}\left(\omega_{a i}^{2} \Delta \mathbf{M}-\Delta \mathbf{K}\right) \mathbf{a}_{i}}
$$


where $\mathbf{b}$ is a mode shape vector for the initial system, $\mathbf{a}$ is a mode shape vector for the perturbed system, $m$ is the modal mass for the initial system, $T$ is a matrix describing the linear transformation between the two sets of mode shapes, subscript $i$ denotes the regarded mode and subscript $a$ and $b$ denotes the perturbed and initial system respectively. Considering mass change only and rearranging Eq. (5) a term for change in modal mass can be defined as

$$
\Delta m_{i}=\frac{\left(\omega_{b i}^{2}-\omega_{a i}^{2}\right) T_{i i}}{\omega_{a i}^{2}} m_{i}=\mathbf{b}_{i}^{T} \Delta \mathbf{M} \mathbf{a}_{i}
$$

By repeating the operation of subjecting the system with unit mass changes in all DOF's, the corresponding modal mass change is determined. This procedure is conducted by means of the right-hand side of Eq. (6) and is repeated for all mode shapes. Hereby, a row vector for each mode can be constructed. This vector contains information on how the modal mass of a specific mode will vary due to sequential mass change in all the DOF's of the system. Therefore, the observed change $\Delta m_{i}$ can be expressed as

$$
\Delta m_{i}=\Delta \mathbf{m}_{i}^{T} \Delta \mathbf{u}_{M}
$$

where $\Delta \mathbf{u}$ is the change vector. The observed modal mass change can be calculated by means of the middle term in Eq. (6) hence the change vector becomes the only unknown of Eq. (7). A similar equation for stiffness changes can be reached by excluding the mass change matrix in Eq. (5) and following the same approach.

\subsection{Final System of Equations}

By combining the derived expressions based on the mode shape sensitivities and the Bernal Projection equation regarding mass change, i.e. Eq. (4) and Eq. (7), a system of equations can be obtained. For mode $i$ we obtain

$$
\left\{\begin{array}{c}
\Delta m_{i} \\
\Delta \mathbf{b}_{i}
\end{array}\right\}=\left\{\begin{array}{c}
\Delta \mathbf{m}_{i}^{T} \\
\Delta \mathbf{B}_{M, i}
\end{array}\right\} \Delta \mathbf{u}_{M}
$$

Hence, Eq. (8) utilizes the observed changes in both frequencies and mode shapes hereby drawing more information into the process of determining the damage vector and ultimately increasing the robustness of the technique. Eq. (8) can be extended to cover changes in stiffness by inserting the stiffness versions of Eq. (4) and (7). For mode $i$ the final system of equations will read

$$
\left\{\begin{array}{c}
\Delta m_{i} \\
\Delta k_{i} \\
\Delta \mathbf{b}_{i}
\end{array}\right\}=\left[\begin{array}{cc}
\Delta \mathbf{m}_{i}^{T} & {[0]} \\
{[0]} & \Delta \mathbf{k}_{i}^{T} \\
\Delta \mathbf{B}_{M, i} & \Delta \mathbf{B}_{K, i}
\end{array}\right]\left\{\begin{array}{c}
\Delta \mathbf{u}_{M} \\
\Delta \mathbf{u}_{K}
\end{array}\right\}
$$

where $\Delta k$ is the observed change in modal stiffness, $\Delta \mathbf{k}$ is the sensitivity vector and subscript $K$ denotes the case of stiffness change. The vector on the left-hand side is defined as the change vector $\Delta \mathbf{c}_{\boldsymbol{i}}$ and the vector on the right-hand side is defined as the damage vector $\Delta \mathbf{u}$. Finally, the matrix in Eq. (9) is defined as the combined sensitivity matrix $\mathbf{S}_{i}$. Thus, Eq. (9) can be expressed as

$$
\Delta \mathbf{c}_{i}=\mathbf{S}_{i} \Delta \mathbf{u}
$$

So for all modes of the system the damage vector is found as

$$
\Delta \mathbf{u}=\mathbf{S}^{+} \Delta \mathbf{c}
$$




\section{CASE STUDY: GLASS PLATE}

In this section the theory described above is utilized in a case study of an FE model of a rectangular glass plate. The data gathered in the simulation are results of a dynamic analysis routine from FE software executed before and after mass perturbation. The main focus has been centered on mass change detection for several reasons but primarily due to the fact that the computational routines regarding mass detection are less burdensome.

The simulation procedure; Initially, the mode shapes and frequencies of the undisturbed system are used to build up the sensitivity matrix. Secondly, the system is subjected to either mass or stiffness alterations, then the modal properties are extracted from the "damaged" system. Finally, the damage vector is determined by multiplying the calculated change in modal properties with the sensitivity matrix in question.

\subsection{Finite Element Model}

The dynamic characteristics of the glass plate are computed by means of a FE structure modelled in SDTools [4]. The plate is subdivided into a 3x3 mesh of QUAD4 shell elements resulting in a total of 16 node points. At all node points the rotationaland in-plane degrees of freedom (DOF) are fixed leaving only the out-of-plane DOF's released. Furthermore, the DOF's in 3 of the 4 corners of the plate are retained towards out plane motion thus constituting a dynamic system with 13 DOF's. The figure below shows the nodes and supports of the FE model.

Geometrical properties:

- Length $\quad 1,4 \mathrm{~m}$

- Width $1 \mathrm{~m}$

- Thickness $10 \mathrm{~mm}$

Material properties:

- Young's modulus $70 \mathrm{GPa}$

- Poisson's ratio 0.2

- Density $2548 \mathrm{~kg} / \mathrm{m}^{3}$

- Total weight $\quad 35.672 \mathrm{~kg}$

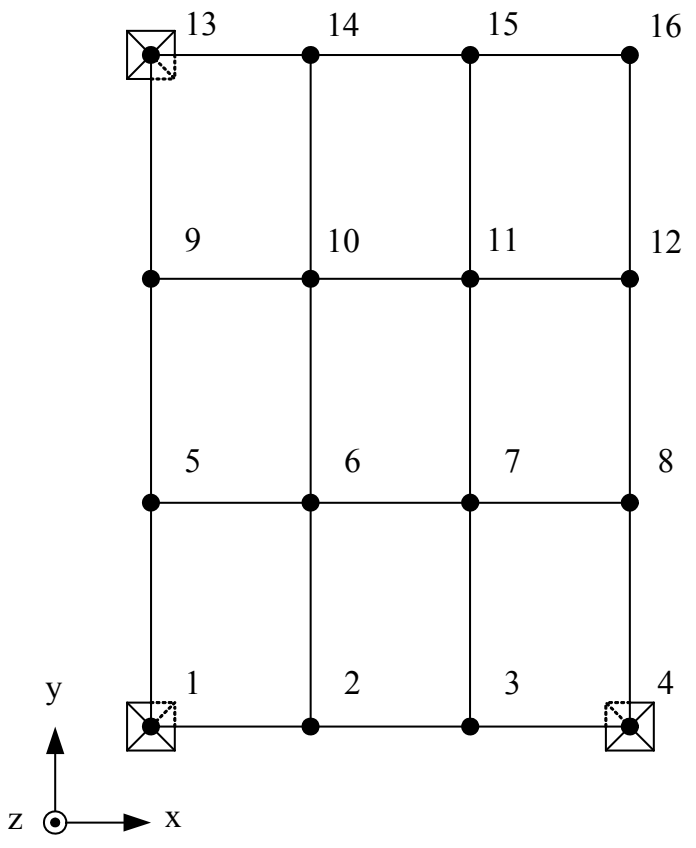

Fig. 1 Glass plate model: nodes and supports

Due to the coarse mesh and the small amount of DOF's the FE model has little resemblance to the properties of a similar physical glass plate, however, the purpose of this study is not to mimic reality but merely to validate the theoretical considerations described in the previous section. 


\subsection{Simulation Results}

In this subsection the results of a series of simulation cases are presented. Besides proving the validity of the method, the purpose is to highlight the extent in which the technique has been tested and display the immediate limitations. All results are displayed in bar plots on a surface resembling the geometry of the FE model.

In figure 2 below a simulation result of a mass change in node 7 is displayed. The size of the mass change is $1 \%$ of the total weight of the glass plate model, $0.357 \mathrm{~kg}$. All 13 modes are used in the calculation of the sensitivity matrix.
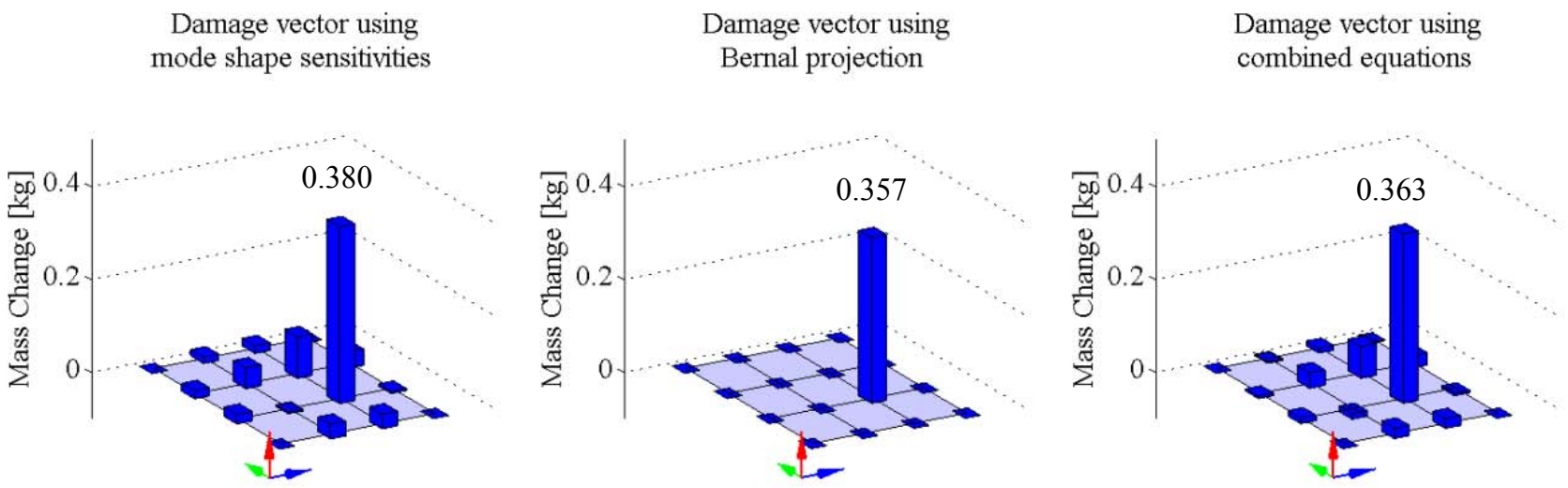

Fig. 2 Damage vectors $1 \%$ mass change in node 7 with 13 modes

The two initial bar plots in figure 2 are the results of Eq. (4) and (7) respectively whereas the outermost right bar plot is the result of the combined equation, i.e. Eq. (11). All three damage vectors clearly indicate a mass change in node 7 along with an accurate estimate of mass size. The damage vector estimated by means of mode shape sensitivities display some scatter in the remaining nodes, this is not unexpected due to the fact that the sensitivity equation is not exact for finite mass changes. In fact the equation will become decreasingly accurate proportional to the increase in value of the mass alteration. On the other hand the damage vector calculated by means of Eq. (7) is exact, because the Bernal projection equation is exact given all modes of the system are utilized.

In Eq. (11) the sensitivity matrix is inverted by means of the Moore-Penrose pseudo inverse. Similar damage detection techniques [1] claims to have issues regarding the amount of mode shapes needed in proportion to the number of DOF's in order to obtain a valid damage vector. Therefore, a study with a decreasing number of modes used in the calculation of the sensitivity matrix has been undertaken. Figure 3 displays the results from a simulation where only 7 of the modes are used in the calculation of the sensitivity matrix.
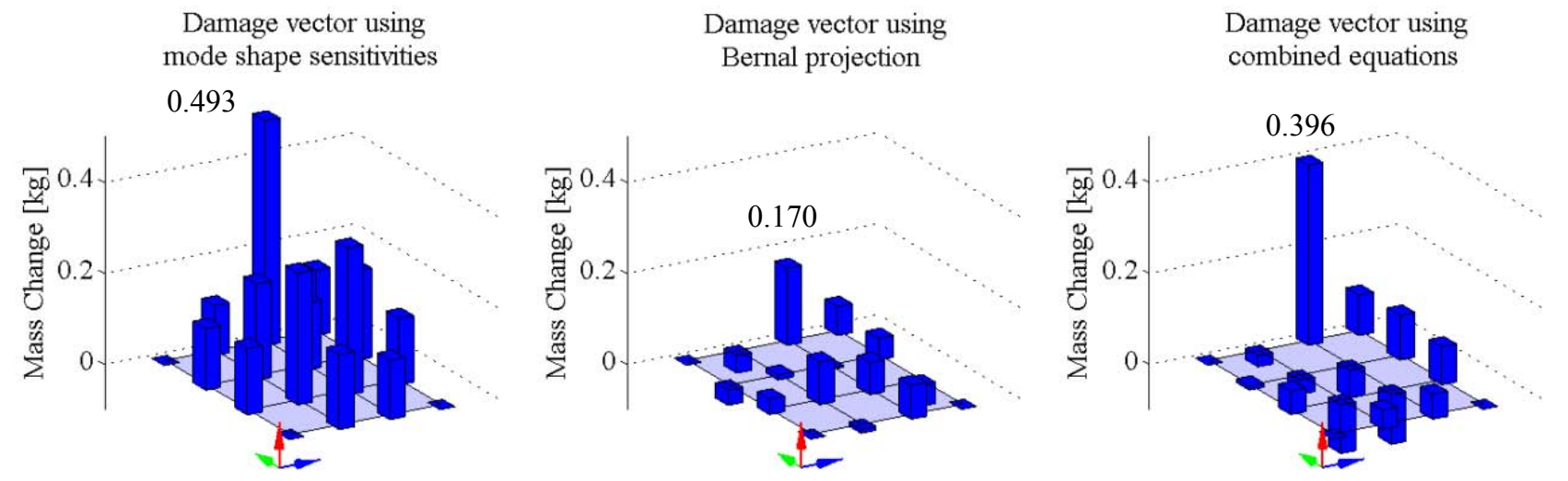

Fig. 3 Damage vectors $1 \%$ mass change in node 15 with 7 modes

The lack of information from the higher modes clearly affects the resulting damage vectors which is evident from the plots in figure 3. The damage vectors shown in figure 3 are not as distinct as the plots in figure 2 and suggest mass changes in other locations than the position of the actual mass change. The source of this problem lies in the inversion of the sensitivity matrix 
which has been clarified by researchers working with similar methods [5] and a solution using a so-called "weighted" pseudo-inverse has been suggested. Another important note regarding figure 3 is that the combination of the mode shape sensitivity and Bernal projection equation results in a sensitivity matrix holding more information and therefore leads to an improved damage vector.

\section{Damage vector using} mode shape sensitivities

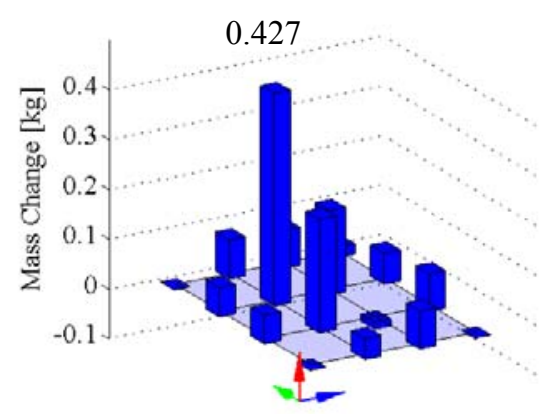

Damage vector using Bernal projection

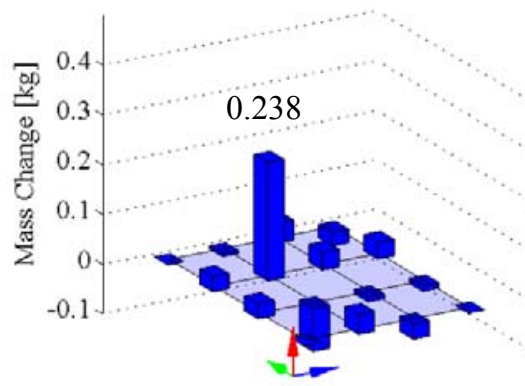

Damage vector using combined equations

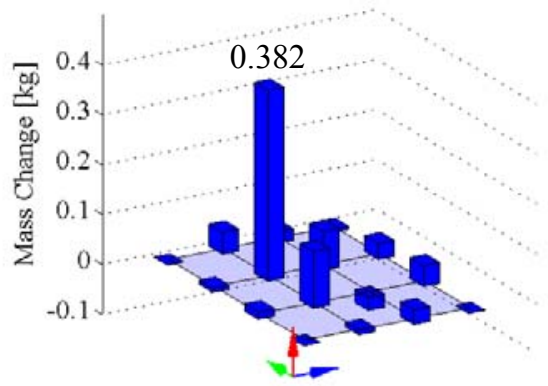

Fig. 4 1\% Mass change in node 10, 10 modes, 0.1\% noise on frequencies and 2\% noise on mode shapes

In the final simulation presented in this paper the frequencies and mode shape has been polluted with noise in the range of what can be expected from real measured data in order to create a realistic dataset. The solution using the combined equation, shown in the outermost right plot in figure 4 , is proven to be quite robust even if the identified modal parameters contain noise.

\section{CONCLUSIONS AND FUTURE WORK}

The result of the simulations presented in this paper clearly states that the proposed method can detect mass change, position and magnitude, by using modal properties. Furthermore the technique exhibits sturdiness towards noise. The inverse issue will become the main subject of interest in the ongoing research.

Due to practical reasons the mass detecting part of the technique, at this point, has been tested more extensively than the stiffness detecting part. The latter part, however, is expected to perform just as well as mass detecting part - at this point there is no immediate circumstance that suggests the opposite.

\section{REFERENCES}

[1] Parloo, E., Guillaume, P., Van Overmeire, M., Damage assessment using mode shape sensitivities, Mechanical Systems and Signal Processing, 17 (3), 499-518, 2003

[2] Bernal, D., Modal scaling from known mass perturbations, Journal of Engineering Mechanics, 130 (9), 1083-1088, 2004

[3] Heylen, R.B., Lammens, S. and Sas, P., Modal Analysis Theory and Testing, Katholieke Universiteit Leuven, Faculty of Eng., Dept. of Mech. Eng., 1997

[4] SDTools - vibration software and consulting, www.sdtools.com

[5] Parloo, E., Vanlanduit, S., Guillaume, P., Verboven, P., Increased reliability of reference-based damage identification techniques by using output-only data, Journal of Sound and Vibration, 270, 813-832, 2004 